\title{
A Dynamic Control Method for Signalized Intersections Using the Flow Fluctuation Amplitude
}

\author{
Jiayu Hang $\mathbb{D}$, Xizhao Zhou $\mathbb{D}$, and Jiawen Wang $\mathbb{D}$ \\ Business School, University of Shanghai for Science and Technology, Shanghai 200093, China \\ Correspondence should be addressed to Xizhao Zhou; xzzhou@usst.edu.cn
}

Received 16 July 2020; Revised 31 August 2020; Accepted 7 October 2020; Published 23 October 2020

Academic Editor: Giancarlo Consolo

Copyright ( $\odot 2020$ Jiayu Hang et al. This is an open access article distributed under the Creative Commons Attribution License, which permits unrestricted use, distribution, and reproduction in any medium, provided the original work is properly cited.

\begin{abstract}
Intersections are a vital component of the urban road network. Their use is essential for traffic efficiency and operational stability. In this paper, a dynamic control method for two-phase signal control intersection is proposed by using the intercycle flow standard deviation. This technique can dynamically assign weights to different control goals of traffic efficiency and operational stability. For intersections with large flow fluctuation amplitudes, greater weight is given to the control goal of operational stability to reduce the sensitivity of the signal control on flow fluctuation. For intersections with a small flow fluctuation amplitude, greater weight is given to the control goal of traffic efficiency to maximize the intersection throughput. Compared with the plan selection and pretimed control method, the numerical results can validate that this method has better control performance under different flow fluctuations amplitude. The fluctuation range length is significantly optimized, especially in the cases of the low and medium flow saturation conditions.
\end{abstract}

\section{Introduction}

It is generally accepted that the efficient management of traffic flow to utilize traffic capacity is an essential objective of traffic signal control. Originally, most signal control intersections were operated by the time-of-day fixed-time control method, which assumes that traffic demand remains constant [1]. Previous studies usually presumed that the arrival rate is constant through the day and that the input traffic flow is the average traffic flow or the maximum traffic flow over a period of time [2-4]. In real-world conditions, traffic demand significantly fluctuates over time. Traffic flow may vary greatly even at the same time of the day or the same day of the week $[5,6]$. If only a fixed flow is used as the timing basis, the timing scheme will be susceptible to flow fluctuation, resulting in poor control reliability [7]. Analyzing traffic flow fluctuation on signal control has become a current area of research.

A number of authors have considered the effects of flow fluctuations on traffic control issues. Research on traffic fluctuation at intersections can be divided into two types: (i) Signal optimization considering flow fluctuation. This method usually assumes that traffic flow obeys a random distribution for representing demand fluctuations [8-11]. However, further analysis on the mechanism of flow fluctuation amplitude on the signal control, such as the factors of mean and variance of the random distribution for traffic flow, is often overlooked.

(ii) Evaluation of intersections' operational performance that considers flow fluctuations. Relevant research mainly focuses on the signal control performance under different demand fluctuations but does not further consider how to use the influence of flow fluctuations to optimize performance.

To fill the above-mentioned gaps, this paper proposes a dynamic control method for a two-phase signal control intersection. The standard deviation of traffic flow is used as an input parameter for representing the flow fluctuation amplitude. This proposed method will be compared with the timing control and plan selection control method. Then, the applicability of this proposed method will be analyzed. 
The contributions of this paper can be summarized by the following two perspectives:

(i) The phenomenon that flow fluctuation exists during signal cycles. For the first time, the influence of timevarying traffic flow on signal control intersection is studied from the perspective of intercycle flow fluctuation. The standard deviation of traffic flow may be the first to be used to represent flow fluctuation amplitude.

(ii) A dynamic control method is proposed for a twophase signal control intersection. The proposed dynamic model considers both traffic efficiency and operational stability of the intersection according to the standard deviation of traffic flow.

Following this introduction, the remainder of this paper is arranged as follows: Section 2 presents a literature review. Section 3 gives an introduction on dynamic control method for intersection. Section 4 proposes the dynamic control model considering flow fluctuation amplitude. Section 5 is the comparison between the proposed control method and signal timing control and the plan selection control method. In addition, the applicability analysis of dynamic control method is given. Section 6 presents the conclusion and discussion on future research directions.

\section{Literature Review}

To date, several studies have focused on the optimization of the intersection signal control using the flow fluctuation amplitude. A multi-objective optimization model using traffic flow fluctuations has been constructed for single pretimed-controlled intersections by Zhang et al. [8]. The first layer of that model aims to improve the intersection throughput and the second layer attempts to improve the stability of signal control. Results show that that effectively reduces the average delay and improves the robustness of signal control. However, it only analyzes in 10-minute units. Heidemann et al. conducted a series of trials in which they assumed that vehicles, which arrive in a given time interval, follow a Poisson arrival process and that the number of vehicles arriving in different but equal time intervals is the same and is independently distributed. However, they did not study the flow fluctuation problem from the perspective of a signal period, nor did they consider the completely different flow distribution in different consecutive time intervals [9]. Han proposed an approach for optimizing traffic signal setting by handling time-varying demands, where the overall analysis period (usually one hour) is divided into a sequence of subperiods (usually between 5 and $15 \mathrm{~min}$ ). In that model, traffic was constant throughout all subperiods [10]. Chen et al. proposed a robust signal control model under the condition of traffic fluctuations. The fluctuation of traffic in their paper was considered from three different aspects: the distribution of traffic demand, the distribution of base saturation flow rate, and the distribution of actual travel speed [11].

To date, several studies have focused on the influences of flow fluctuations on signal control intersection performance.
In an analysis on evaluation of signalized intersection performance using flow fluctuation amplitude, Lee Han et al. studied the effects of demand fluctuations on vehicle delays at the signalized intersections. They reported that delay model based on Webster often regards that demand fluctuation during the whole analysis period (usually 15 minutes or more) as the same. As a result, the delay estimation at intersections, where demand fluctuation causes obvious traffic flow fluctuation, is often lower than the actual value. Their research shows that demand fluctuation pattern during different periods leads to different vehicle average delay. In other words, the demand fluctuation from low to high causes a higher average vehicle delay than the pattern from high to low, even if the overall demand is exactly the same [5]. Olszewski et al. proposed a coefficient of variation of 5-minute volumes as a convenient measure of flow variability. They developed a simulation model to study the effect of fluctuations on signal performance. The relationship between the degree of saturation, volume variability, and average delay was studied for a range of input parameters [12].

In all those studies, flow fluctuation is recognized as important for both signal control and performance measurement at intersections. One can find that the previous research mainly analyzed flow fluctuation during a period of time and ignored the influence of intercycle flow fluctuation amplitude (standard deviation).

\section{Methodology}

3.1. Preliminary. Commonly used traffic flow distribution functions include negative exponential distribution [13], shifted negative exponential distribution [14], Erlang distribution [15], and Cowan's M3 headway distribution [16]. When traffic flow is assumed to obey these distributions, the mean and standard deviation of which are fixed. Under the condition of time-varying traffic flow, most traffic flow models allow the traffic flow rate to change once in an hour. Some models allow changes at every 15 minutes. A few models allow a change of traffic flow rate once every five minutes, but few models vary once every minute [5]. However, the effect of varying traffic rates has not been studied. In order to show whether this variation is important, traffic data of an actual intersection has been collected. The flow data is of the intersection of Nanjing middle road and Henan middle road in Yangpu District of Shanghai. Then, the intercycle flow standard deviation is calculated every 5 cycles. As shown in Figure 1, the standard deviation of traffic flow varies between 2 and 10, which shows that the flow fluctuation phenomenon exists in intercycles.

3.2. Problem Description. When analyzing traffic flow's time headway distribution, many previous studies used the standard deviation of the speed differences between following cars. In that way, the different regimes can be identified [14, 17]. In this paper, we use the intercycle standard deviation of traffic flow, $D(t)$, to represent the flow fluctuation amplitude. The larger the value of $D(t)$, the 


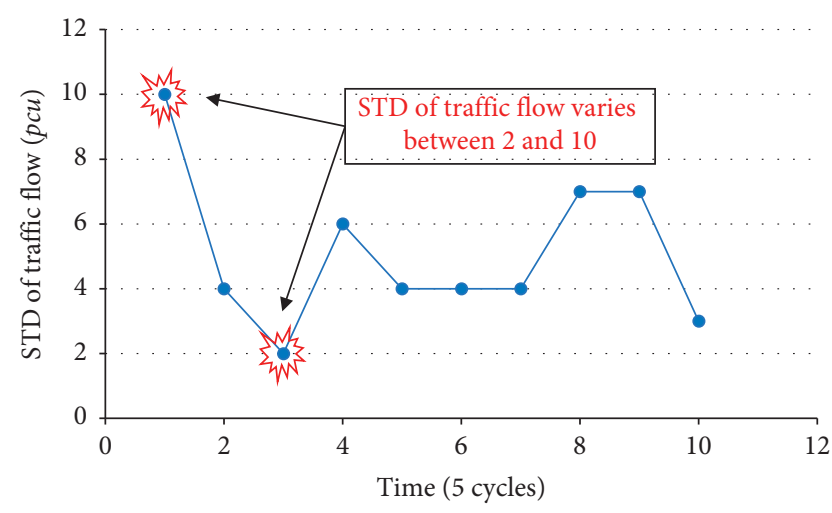

FIgURE 1: The standard deviation of traffic flow.

greater the flow fluctuation amplitude during a continuous period of time. Where $Q$ is traffic matrix and $n$ is the number of cycles, the traffic flow can be expressed as $Q=\left[q_{1}, q_{2}, q_{3}, \ldots, q_{n}\right]$ and the average traffic flow is $E(Q)$. The intercycle standard deviation of traffic flow, $D(t)$, is defined as $D(t)=\sqrt{(1 / n) \sum_{w=1}^{n}\left(q_{w}-E(Q)\right)^{2}}$.

For each transportation facility, there is a prescribed method for estimating the capacity and the service level. In addition, there are some performance indicators that can be calculated, which reflect on the operation of these transportation facilities under the given traffic and control conditions [18]. In this paper, a dynamic control model is proposed for a two-phase signal control intersection, which dynamically optimizes the two different performance indicators. These indicators represent traffic efficiency and the operational stability of the intersection. The standard deviation of delay $\sigma(d)$ is selected to measure operational stability, while the queue length $L$ is used to reflect intersection efficiency. Intersections with large flow standard deviations obtain greater weight for the operational stability so that the stability control objective reduces the sensitivity of signal control to flow fluctuations. For intersections with small flow standard deviations, a greater weight is given to the efficiency control objective by reducing the queue length as much as possible.

Wide fluctuations of traffic flow will affect the performance of traffic signals, as queueing is more likely to occur and therefore a random delay increases. In this case, optimizing the queue length at the approach road can reduce the vehicle stop delay, which will improve the traffic efficiency of the intersection. Therefore, the queue length is used to reflect the intersection efficiency. At a signal control intersection, a certain queue length is allowed under certain conditions [19-21]. Hence, it is essential to set an expected queue length. When the queue length at the approach road is greater than the expected queue length, the signal timing plan should be optimized to increase the intersection throughput and reduce the queue length gradually. A flowchart of the dynamic control method is given in Figure 2.

The left part of the flowchart gives an introduction on the process of dynamic control, including the crucial parameters to be calculated. The right part shows the proposed control scheme. In this study, the time horizon is divided into several discrete time intervals, and each time interval is indexed by $n t_{c}$. For each time interval $n t_{c}$, the signal control is fulfilled by three stages: perception, decision-making, and execution. In the perception stage, the data such as the traffic flow, current signal timing plan, actual queue length at approach roads of the intersection, and the traffic flow standard deviation is obtained. In the decision-making period, whether the current signal control strategy meets the control objective is judged. In this study, it refers to whether the actual queue length is more than the expected queue length. If it is more than the expected queue length, the dynamic control strategy is implemented to adjust the control objectives according to flow fluctuation amplitude, and new signal parameters are calculated. If the actual queue length is not more than the expected queue length, the stability control objective is optimized only. Finally, in the execution stage, the traffic light will carry out the new signal timing plan.

3.3. Dynamic Control Model considering Flow Fluctuation Amplitude. To facilitate the model formulation, the key notations used are summarized in Table 1.

3.3.1. Queue Length at the Approach Road and Delay Standard Deviation. According to the dynamic control flowchart, it is necessary to calculate the vehicle queue length and standard deviation of vehicle delay before creating a dynamic control model for the signalized intersections.

To create the formula, the time $t$ is used as integer multiple of signal cycles in this paper. The queue length is calculated at the end of every $n$ cycles. The traffic flow of $i$ approach road in cycle $j$ is defined as $q_{i}^{j}$. Subsequently, the queue length of approach No. 1 and approach No. 2 (see Figure 3 ) at time $t$ can be calculated by

$$
\begin{aligned}
& l_{1}(t)=\sum_{j}^{j+n} q_{1}^{j} t_{c}-n s x_{1 g}, \\
& l_{2}(t)=\sum_{j}^{j+n} q_{2}^{j} t_{c}-n s x_{2 g} .
\end{aligned}
$$

In this paper, the standard deviation of delay $\sigma(d)$ is selected to measure operational stability. The mesoscopic model is utilized for the derivation of $\sigma(d)$. For the delay on an isolated intersection, the distribution $P(d)$ of the delay, $d$, is given by [22]

$$
P(d)=\alpha \delta(d)+\beta, \quad 0<d<x_{i r},
$$

where $\alpha=1-x_{i r} /\left\{t_{c}\left(1-\left(q_{i} / s\right)\right)\right\}$ and $\beta=\left\{t_{c}\left(1-\left(q_{i} / s\right)\right)\right\}^{-1}$.

In this model, vehicles arrive uniformly in each cycle. For this uniform delay distribution, the expectation value and the standard deviation can be calculated by the following equations: 


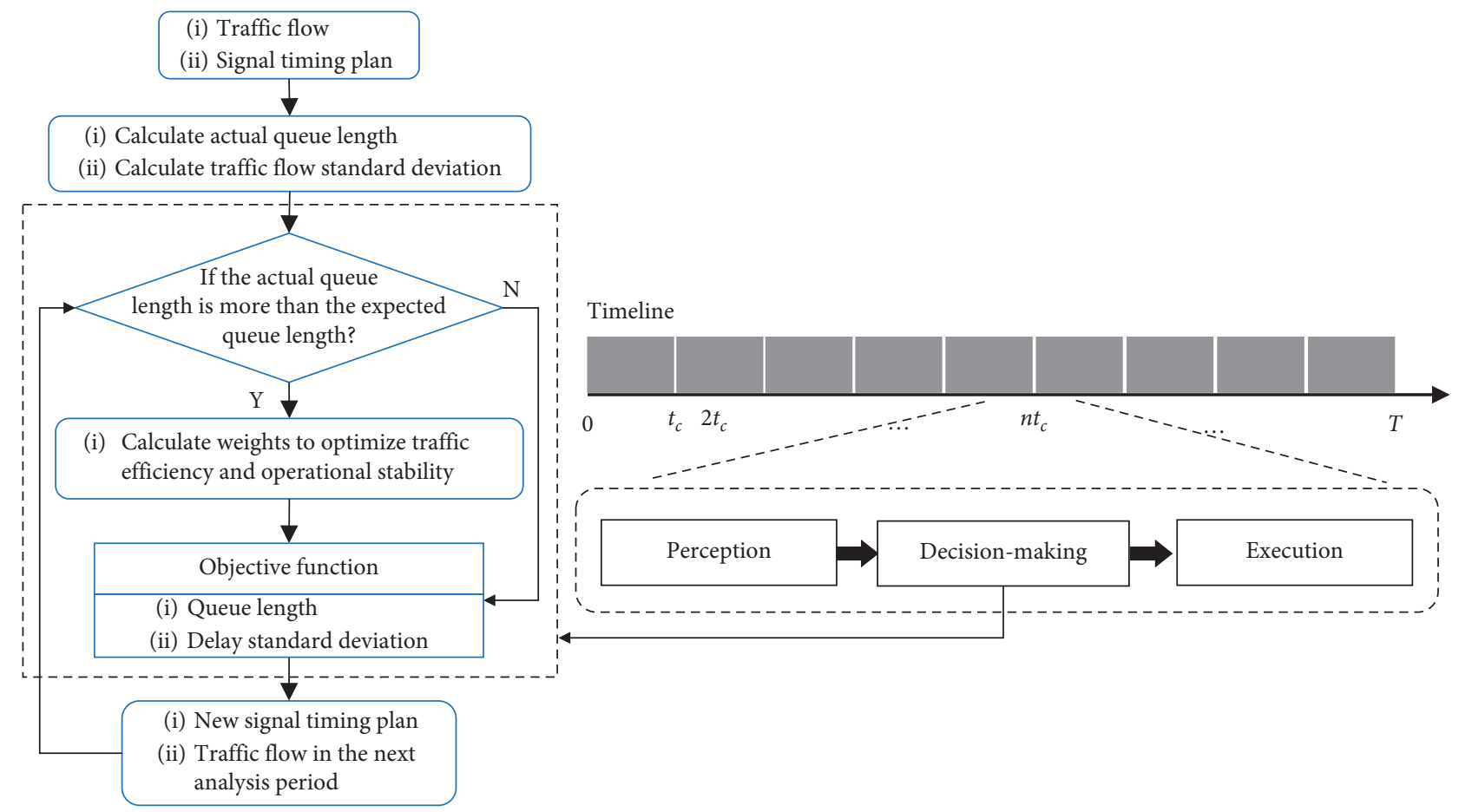

FIgUre 2: The flowchart of the dynamic control method.

TABle 1: Parameter descriptions.

\begin{tabular}{|c|c|}
\hline$t$ & Time, $s$ \\
\hline$t_{c}$ & Cycle length, $s$ \\
\hline$x_{\mathrm{ig}}$ & Green time of $i$ approach road, $s$ \\
\hline$x_{\mathrm{ig} \min }$ & The minimum green time of $i$ approach road, $s$ \\
\hline$x_{\mathrm{ir}}$ & Red time of $i$ approach road, $s$ \\
\hline$q_{i}$ & Traffic flow of $i$ approach road, $\mathrm{pcu} / \mathrm{h}, \mathrm{pcu}=$ passenger car unit \\
\hline$s$ & Saturation flow, pcu/h \\
\hline$\Delta t$ & Every analysis period of signal optimization, $s$ \\
\hline$L_{i}$ & Expected queue length of $i$ approach road, $m$ \\
\hline$l_{i}(t)$ & Actual queue length of $i$ approach road, $m$ \\
\hline$l_{\max }^{i}$ & The maximum queue length of $i$ approach road, $m$ \\
\hline$e(t)$ & Deviation matrix of actual queue length and expected queue length \\
\hline$D(t)$ & Standard deviation of traffic flow during 5 cycles \\
\hline$D$ & The maximum standard deviation of traffic flow \\
\hline$\eta_{1}, \eta_{2}$ & The weight given to optimization objective, $\eta_{1}+\eta_{2}=1$ \\
\hline$E(d)$ & The expectation value of delay, $s$ \\
\hline$\sigma(d)$ & Standard deviation of delay during 5 cycles \\
\hline$L(\cdot)$ & Objective function \\
\hline
\end{tabular}




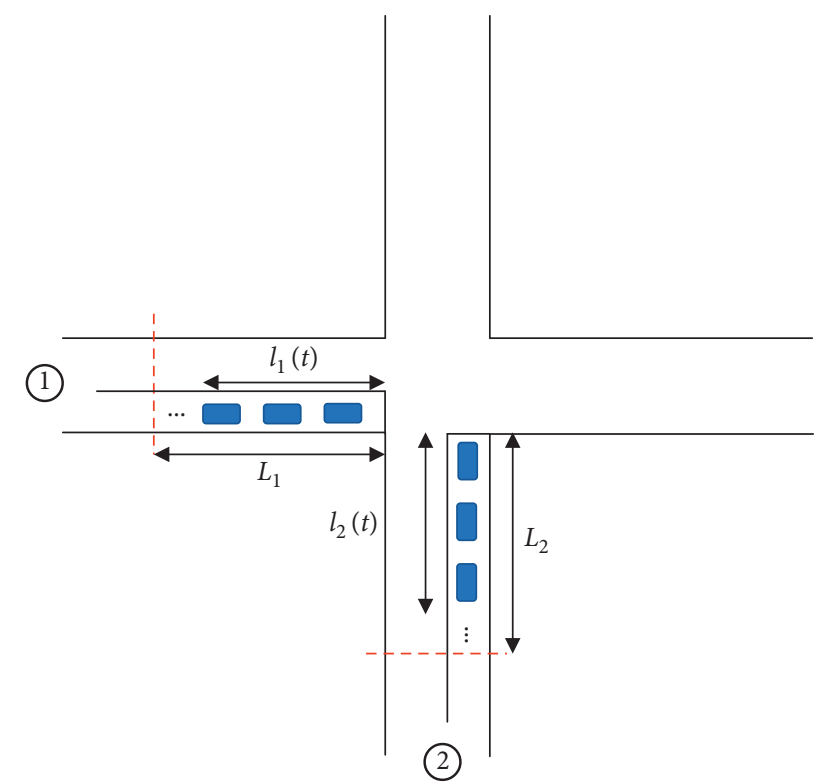

Figure 3: An intersection layout considering queue length constraints.

$$
\begin{aligned}
E(d) & =\int_{0}^{x_{i r}} \mathrm{~d} P(d) d(d)=\frac{x_{i r}^{2}}{2 t_{c}\left(1-\left(q_{i} / s\right)\right)} \\
\sigma(d) & =\sqrt{E\left(d^{2}\right)-E^{2}(d)} \\
E\left(d^{2}\right) & =\int_{0}^{x_{i r}} \mathrm{~d}^{2} P(d) d(d)=\frac{x_{i r}^{3}}{3 t_{c}\left(1-\left(q_{i} / s\right)\right)} \\
\sigma(d) & =E(d) \sqrt{\frac{4 t_{c}\left(1-\left(q_{i} / s\right)\right)}{3 x_{i r}}-1}=E(d) \sqrt{\frac{2 x_{i r}}{3 E(d)}-1 .}
\end{aligned}
$$

\subsubsection{Dynamic Control Method for Signal Control} Intersection. The actual queue length calculated in the previous section is compared with the expected queue length. The expected queue length refers to the maximum queue length allowed at the approach road of the intersection without affecting the upstream intersection. When the actual queue length is more than the expected, the operation of the intersection may affect the upstream intersection due to the impact of traffic flow fluctuations. Deviation matrix $e(t)$ is used to represent the comparison results. $e(t)$ can be obtained from the following definitions:

$$
e(t)= \begin{cases}{[1,1],} & l_{1}(t)>L_{1}, l_{2}(t)>L_{2}, \\ {[1,0],} & l_{1}(t)>L_{1}, l_{2}(t) \leq L_{2}, \\ {[0,1],} & l_{1}(t) \leq L_{1}, l_{2}(t)>L_{2}, \\ {[0,0],} & l_{1}(t) \leq L_{1}, l_{2}(t) \leq L_{2} .\end{cases}
$$

If $e(t)$ is equal to $[1,1]$, the priority will be given to optimize the green time ratio at the approach road with a longer queue length. If $e(t)$ is equal to $[1,0]$ or $[0,1]$, the signal cycle is unchanged; then, the green time ratio will be changed to increase the green time of the approach road where the actual queue length is more than the expected queue length. If $e(t)$ is equal to [0,0], then there is no need to optimize signal timing to reduce the queue length of the approach road.

In addition to considering the optimization goal of queue length, the proposed model also takes the delay standard deviation into account according to the intercycle flow standard deviation. The dynamic control function that simultaneously considers traffic efficiency and operational stability performance indicators uses the following formula:

$$
\begin{aligned}
\min L\left(x_{i g}, D(t)\right)= & \eta_{1} e(t)\left(\begin{array}{c}
l_{1}(t) \\
l_{2}(t)
\end{array}\right)+\eta_{2} \sigma(d), \\
\text { s.t. }= & \left\{\begin{array}{l}
\eta_{2}=\min \left(1, \frac{D(t)}{D}\right), \\
\eta_{1}=1-\eta_{2} \\
x_{\mathrm{ig}}+t_{\mathrm{lost}}=t_{c}, \\
l_{i}(t) \leq l_{\text {max }}^{i}, \\
L_{i} \leq l_{\text {max }}^{i}, \\
x_{\text {ig }}>x_{\text {ig } \min } .
\end{array}\right.
\end{aligned}
$$

The maximum standard deviation of traffic flow $D$ is predetermined. In these equations, $\eta_{1}$ is the weight to optimize traffic efficiency and $\eta_{2}$ is the weight to optimize operational stability.

3.3.3. Sensitivity Analysis on the Maximum Standard Deviation of Traffic Flow D. When the traffic efficiency and operational stability of intersection are dynamically controlled with different weights, the intercycle flow standard deviation is used as an input parameter to compare with the maximum flow standard deviation. Hence, it is essential to predetermine $D$. A sensitivity analysis on $D$ is conducted in Figure 4.

When the saturation is more than 0.3 , the queue length at the approach road becomes noticeable. Hence, this paper only discusses when saturation is greater than 0.3 . Figure 4(a) shows the queue length under different flow fluctuations when the saturation degree is from 0.3 to 0.6. The saturation degree varies between 0.3 and 0.6 , and the maximum saturation degree is less than or equal to 0.6 . The queue length will be smallest when the maximum intercycle flow standard deviation is 13 , as shown in the red box of Figure 4(a). Figure 4(b) shows the queue length under different flow fluctuations when the saturation degree is from 0.6 to 0.9 . The saturation degree varies between 0.6 and 0.9 , and the minimum saturation degree is more than 0.6. 


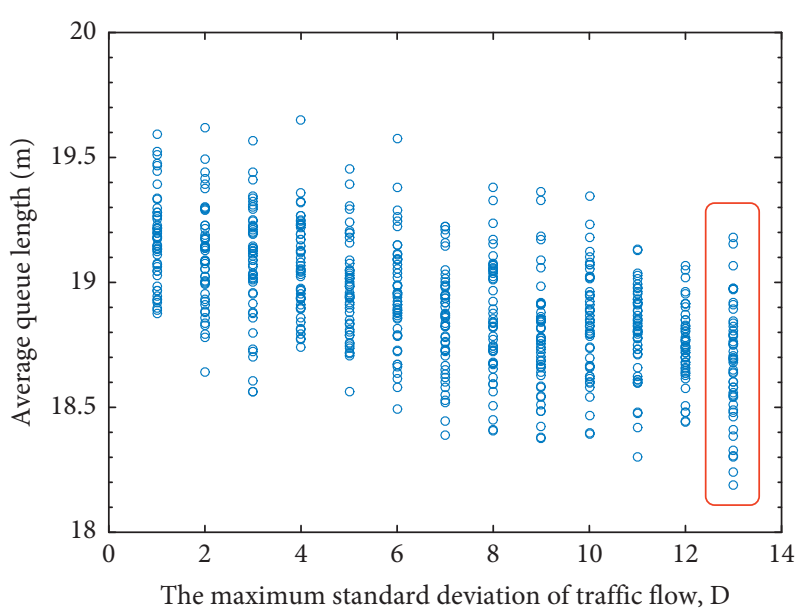

(a)

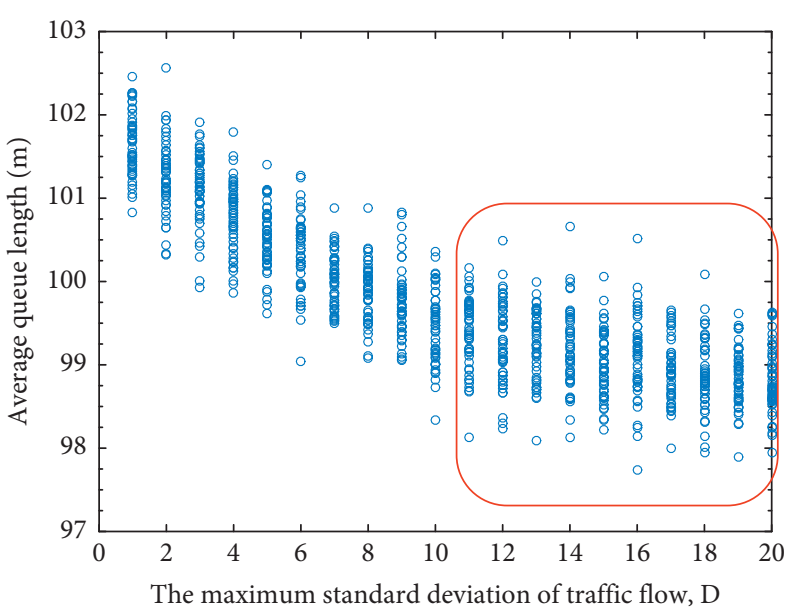

(b)

FIgUre 4: Different standard deviations of traffic flow $D$ corresponding to average queue length under different saturations. (a) Saturation degree is from 0.3 to 0.6 . (b) Saturation degree is from 0.6 to 0.9 .

The queue length tends to be stable when the maximum intercycle flow standard deviation is more than 11, as shown in the red box of Figure 4(b). Therefore, the maximum intercycle flow standard deviation, $D$, is set to be 13 when the saturation is between 0.3 and 0.9 .

\section{Results}

4.1. Comparison Analysis of Three Different Signal Control Strategies. In this section, several factors have been considered to investigate their effects on dynamic control. The period of dynamic control is 5 cycles in this paper; i.e., $\Delta t$ is equal to $5 t_{c}$. In the following analysis, the following assumptions have been made:

(1) The number of vehicle arrivals per cycle is a random variable with a known probability distribution. In this study, the normal distribution is employed at the case of an isolated intersection.

(2) In each signal cycle, vehicle arrivals are uniformly distributed.

(3) The saturation flow rate is assumed to be constant.

In this section, the cycle time $t_{c}$ is $90 \mathrm{~s}$ and the saturation flow $\mathrm{s}$ is $1800 \mathrm{pcu} / \mathrm{h}$, and the analysis period of signal optimization $\Delta t$ is $450 \mathrm{~s}$. In addition, the expected queue length of $i$ approach road $L_{i}$ is $150 \mathrm{~m}$ and the maximum queue length of $i$ approach road $l_{m x a}^{i}$ is $180 \mathrm{~m}$. The numerical simulation experiment is carried out with MATLAB, and the experimental results are as follows.

To illustrate the applicability of this section's proposed model, a dynamic control method is proposed for the signal timing plan of a two-phase signal control intersection. Then, the pretimed control and plan selection control method are compared with the proposed method. The Webster method is used to determine the timing plans of pretimed control and plan selection control method. Under the pretimed control strategy, the signal timing plan is obtained based on the maximum $15 \mathrm{~min}$ flow during the peak period. Two signal timing plans are utilized under the plan selection control method. One scheme is calculated based on the maximum $15 \mathrm{~min}$ flow during peak period, and the other is obtained based on $70 \%$ of the maximum 15 min flow during peak period. The performance indicators of standard deviation, average queue length, and average delay are analyzed separately under various saturation with different intercycle flow standard deviation.

4.1.1. The Standard Deviation of Queue Length at an Approach Road. Figure 5 shows that in each flow saturation, as the intercycle flow standard deviation increases, the STD of the generated queue length is increased. The graph shows that the phenomenon of flow fluctuation at the approach road of the intersection has a significant impact on the signal control. When the saturation is low, the STD of queue length, which is caused by dynamic control strategy, is significantly lower than that by the other two control strategies. This effect is shown in a red box. As the saturation continues to rise, the STD of queue length gradually increases. However, it can be seen that the STD of queue length generated by the proposed method is lower than the two control strategies. The red straight line illustrates this phenomenon.

These findings, while preliminary, suggest that the overall impact of the standard deviation of the queue length at the lower mean traffic flow could be considered less crucial than that at the higher mean traffic flow with respect to the efficiency point of view. Moreover, the result obtained for the trend change of the standard deviation of the queue length with respect to the standard deviation of traffic flow shows an expected trend.

4.1.2. The Average Queue Length at an Approach Road. As shown in the red boxes in Figure 6, in the early stages, the growth trend of the average queue length caused is slower. On the whole, the average queue length index of the dynamic 


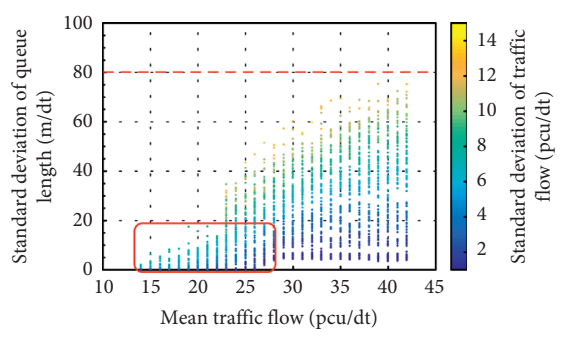

(a)

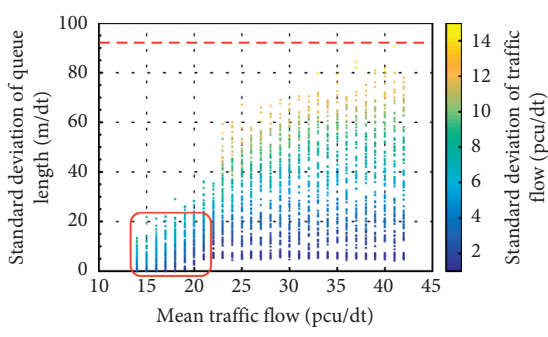

(b)

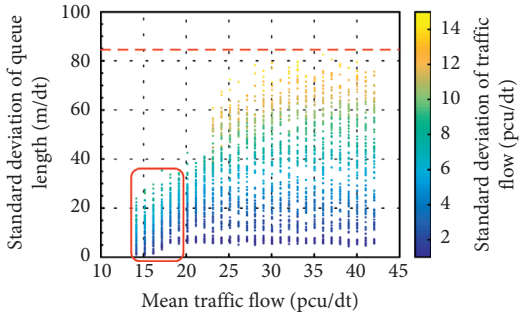

(c)

Figure 5: Standard deviation of queue length under different saturations with different intercycle flow standard deviations. (a) Dynamic control. (b) Plan selection control. (c) Pretimed control.

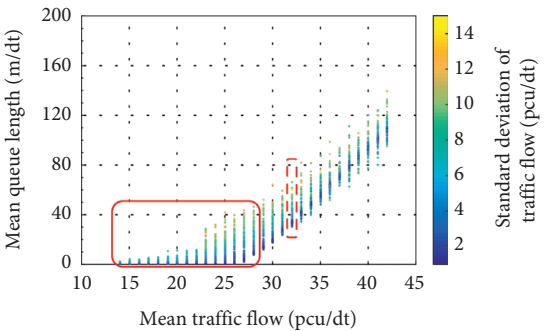

(a)

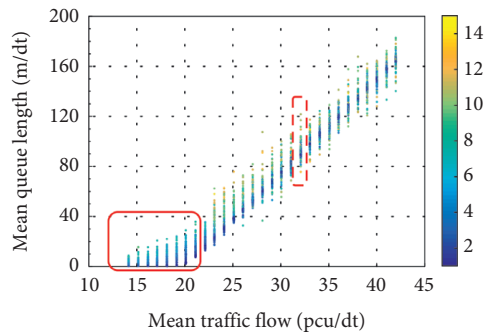

(b)

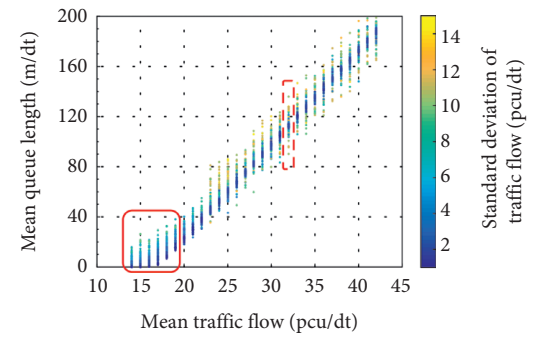

(c)

Figure 6: Average queue length under different saturations with different intercycle flow standard deviations. (a) Dynamic control. (b) Plan selection control. (c) Pretimed control.

control method is better than the other two methods. Compared with the plan selection and pretimed control method, the control strategy can better ensure that the average queue length is smaller when the mean traffic flow is smaller. But under the same circumstances, small flow fluctuations may lead to larger average queue length caused by the other two control strategies, as shown in the dashed box.

\subsubsection{The Average Delay of an Approach Road. As shown in} Figure 7 , the average delay caused by dynamic control strategy is generally lower than that generated by the plan selection and pretimed control strategies. At the same time, as flow saturation continues to increase, the average delay growth trend is more gentle than other methods. Given the differences of the average queue lengths of the methods in Figure 6 , the average delays are expected to be compatible with the respective mean queue lengths.

In summary, these results support the idea of flow fluctuation amplitude having significant impacts on signal control performance at intersections. Hence, it is critical to consider the traffic flow fluctuation when conducting signal control research on urban intersections.

\subsection{Applicability Analysis of the Dynamic Control Method.} In this section, the variation range of intersection performance indicators is analyzed under the dynamic control and plan selection control method. The variation ranges of the three performance indicators (STD of queue length, average queue length, and average delay) are separately calculated. Figure 8 shows the variation ranges under the two control strategies and the optimized percentage of the variation range.

In the previous section, under different saturation degree, the fluctuation range of three performance indicators (standard deviation of queue length, average queue length, and average delay) with different flow fluctuation is obtained. Taking the average queue length as an example, the fluctuation range of average queue length under dynamic control method is $\mathrm{FL}_{d}$, the fluctuation range of average queue length under plan selection control method is $\mathrm{FL}_{p}$, and the optimized percentage is calculated by

$$
\text { optimized perentage }=\frac{\mathrm{FL}_{p}-\mathrm{FL}_{d}}{\mathrm{FL}_{p}} .
$$

As shown in Figure 8(a), the optimization of the variation range in the STD of queue length is apparent. When the intercycle average flow is less than 36 , the optimized percentage is more than $10 \%$. In Figure $8(\mathrm{~b})$, when the intercycle average flow is less than 37 , the optimized percentage of variation range in the queue length is more than $10 \%$. In Figure 8(c), the variation range in the average delay is slight, which is not as obvious as that of the other two indicators. The variation range results on minimum and maximum are summarized in Table 2. 


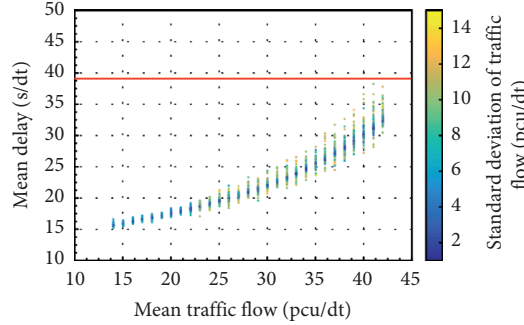

(a)

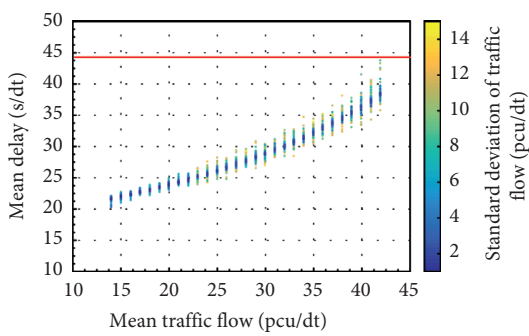

(b)

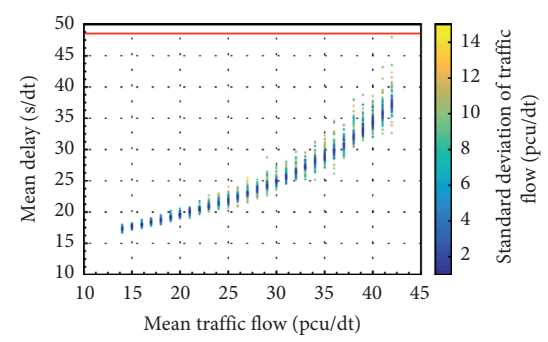

(c)

Figure 7: Average delay under different saturations with different intercycle flow standard deviations. (a) Dynamic control. (b) Plan selection control. (c) Pretimed control.

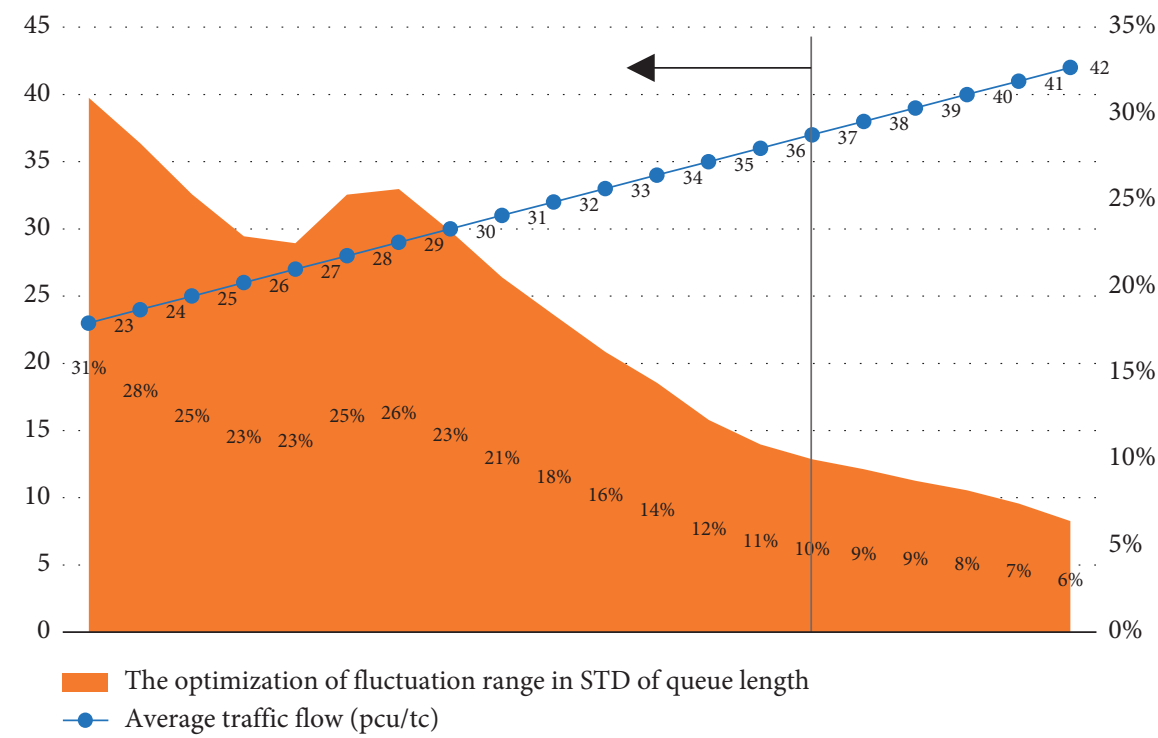

(a)

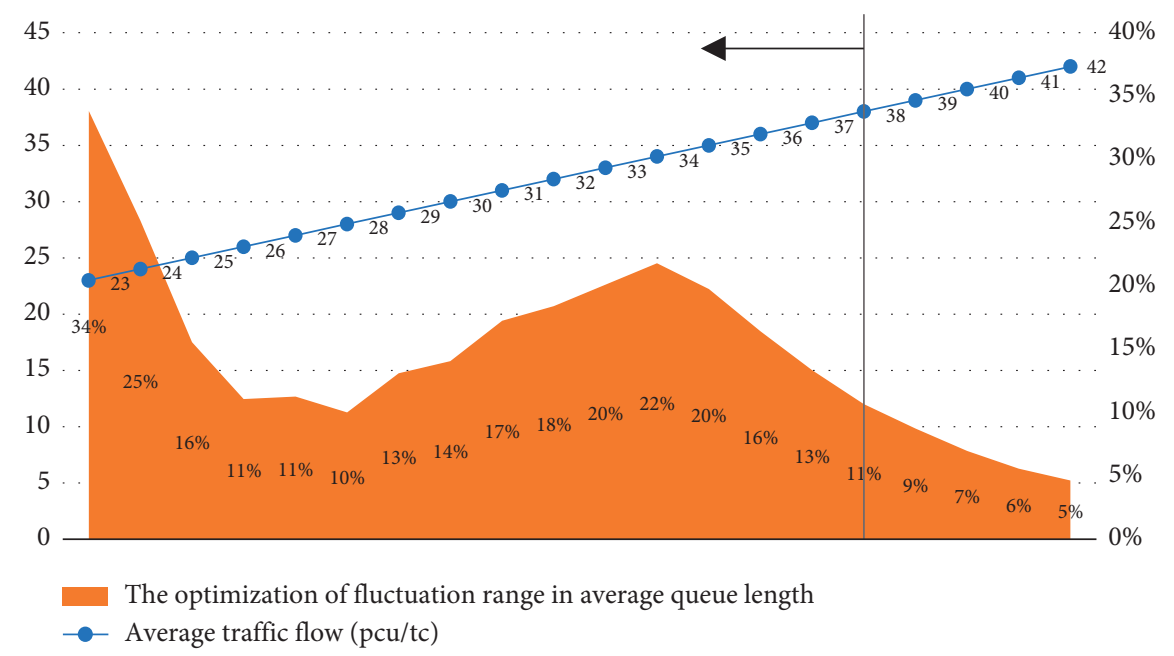

(b)

Figure 8: Continued. 


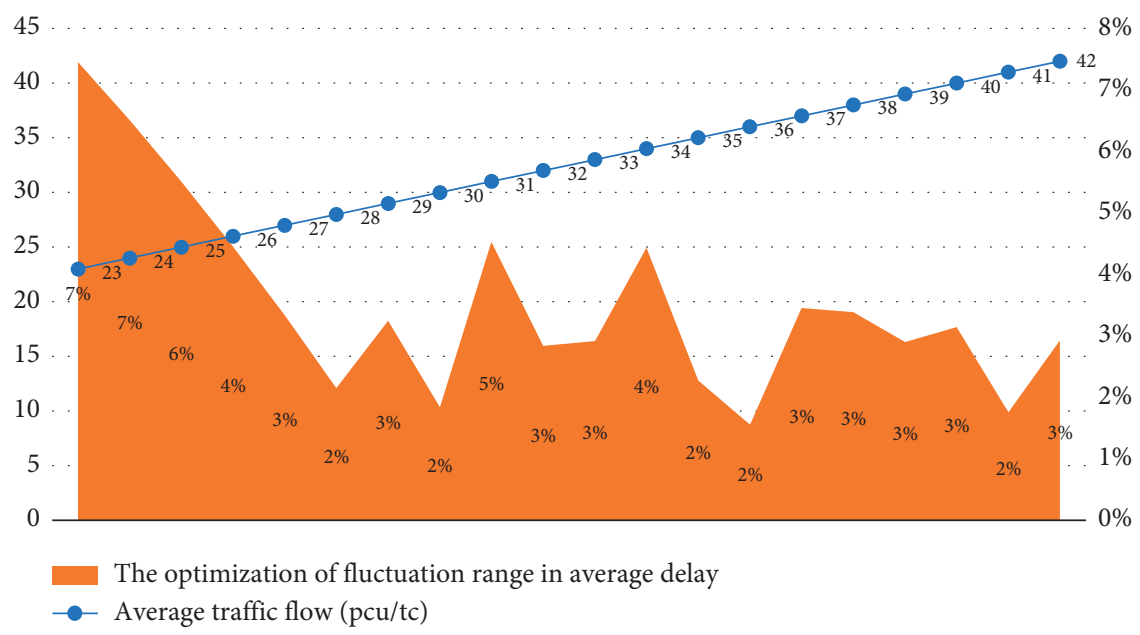

(c)

Figure 8: The optimization of fluctuation range in different performance indicators. (a) The optimization of variation range in standard deviation of queue length. (b) The optimization of variation range in average queue length. (c) The optimization of variation range in average delay.

TABle 2: The minimum and maximum optimized percentage of variation range.

\begin{tabular}{lcc}
\hline Performance indicator & $\begin{array}{c}\text { The optimization of variation } \\
\text { range }\end{array}$ \\
& Minimum (\%) & Maximum (\%) \\
\hline $\begin{array}{l}\text { Standard deviation of queue } \\
\text { length }\end{array}$ & 6 & 31 \\
$\begin{array}{l}\text { Average queue length } \\
\text { Average delay }\end{array}$ & 5 & 34 \\
\hline
\end{tabular}

The present results are significant in at least two major respects:

(i) The results imply that the proposed method can improve the signal control effect under different flow fluctuations amplitude, which would have been much more significant at flow rates close to the saturation.

(ii) The flow fluctuation amplitude at intersections has a significant effect on signal control performance. If the traffic flow saturation is high, the control performance is more sensitive due to the flow fluctuation amplitude. This finding shows the level of robustness of the methods employed at the high saturation levels.

Therefore, it is vital to strengthen the consideration of influences caused by the fluctuation amplitude in traffic flow at intersections.

\section{Conclusions}

In this paper, a dynamic control method for the twophase signal control intersection is proposed, where the standard deviation of intercycle traffic flow is used to represent the flow fluctuation amplitude. On the basis of the intercycle traffic flow standard deviation, the proposed model dynamically adjusts the traffic efficiency and operational stability of the intersection. The dynamic control method is compared with the plan selection and pretimed control method. A numerical study is performed by taking into account various factors (i.e., standard deviation, average queue length, and average delay). In addition, it can be seen from the results that the proposed method has a better control performance than the comparable method under different flow fluctuation amplitudes. Especially in low and medium flow saturation conditions, the fluctuation range of the standard deviation and average queue length is significantly improved.

In summary, it is critical to consider the influence of the flow fluctuation amplitude on the signal control performance at intersections. In this paper, the dynamical control method for two-phase intersection is only considered. It is potentially important to investigate the signal control method for different control types. As only numerical experiments have been carried out in this paper, it is necessary for further verification to use actual data on appropriate simulation experiments and/or conduct suitable field tests.

\section{Disclosure}

The content of this paper reflects the views of the authors, who are responsible for the facts and the accuracy of the data presented herein.

\section{Data Availability}

All the data used to support the findings of this study are included within the article. 


\section{Conflicts of Interest}

The authors declare that they have no conflicts of interest.

\section{Acknowledgments}

Thanks are due to the National Natural Science Foundation of China Project "Research on Key Issues of Reliability Analysis for Random Traffic Network Travel Time" (61273042).

\section{References}

[1] Y. Feng, K. L. Head, S. Khoshmagham, and M. Zamanipour, "A real-time adaptive signal control in a connected vehicle environment," Transportation Research Part C: Emerging Technologies, vol. 55, pp. 460-473, 2015.

[2] S. Araghi, A. Khosravi, and D. Creighton, "A review on computational intelligence methods for controlling traffic signal timing," Expert Systems with Applications, vol. 42, no. 3, pp. 1538-1550, 2015.

[3] M. Aslani, M. S. Mesgari, and M. Wiering, "Adaptive traffic signal control with actor-critic methods in a real-world traffic network with different traffic disruption events," Transportation Research Part C: Emerging Technologies, vol. 85, pp. 732-752, 2017.

[4] Z. Yao, L. Shen, R. Liu, Y. Jiang, and X. Yang, "A dynamic predictive traffic signal control framework in a cross-sectional vehicle infrastructure integration environment," IEEE Transactions on Intelligent Transportation Systems, vol. 21, no. 4, pp. 1455-1466, 2020.

[5] L. D. Han, J.-M. Li, and T. Urbanik, "Impacts of intercycle demand fluctuations on delay," Journal of Transportation Engineering, vol. 135, no. 5, pp. 288-296, 2009.

[6] J. Wang, Y. Wang, M. Yun, and X. Yang, "Development of urban road network traffic state dynamic estimation method," Mathematical Problems in Engineering, vol. 2015, pp. 1-10, Article ID 714149, 2015.

[7] S. Li, C. Wei, X. Yan, L. Ma, D. Chen, and Y. Wang, "A deep adaptive traffic signal controller with long-term planning horizon and spatial-temporal state definition under dynamic traffic fluctuations," IEEE Access, vol. 8, pp. 37087-37104, 2020.

[8] M. M. Zhang, L. Jia, N. Zhou et al., "Robust optimal traffic signal timing of urban signal-point intersection," Journal of Highway and Transportation Research and Development, vol. 28, no. 1, pp. 107-111, 2011.

[9] D. Heidemann, "Queue length and delay distributions at traffic signals," Transportation Research Part B: Methodological, vol. 28, no. 5, pp. 377-389, 1994.

[10] B. Han, "Optimising traffic signal settings for periods of timevarying demand," Transportation Research Part A: Policy and Practice, vol. 30, no. 3, pp. 207-230, 1996.

[11] K. Chen, J. Zhao, V. L. Knoop, and X. Gao, "Robust signal control of exit lanes for left-turn intersections with the consideration of traffic fluctuation," IEEE Access, vol. 8, pp. 42071-42081, 2020.

[12] P. S. Olszewski, "The effect of short time traffic volume fluctuations on traffic signal performance," in Proceedings of the Australian Road Research Board (ARRB) Conference, Adelaide, Australia, 1986.

[13] R. Pueboobpaphan, D. Park, Y. Kim, and S. Choo, "Time headway distribution of probe vehicles on single and multiple lane highways," KSCE Journal of Civil Engineering, vol. 17, no. 4, pp. 824-836, 2013.

[14] A. S. Al-Ghamdi, "Analysis of time headways on urban roads: Case study from Riyadh," Journal of Transportation Engineering, vol. 127, no. 4, pp. 289-294, 2001.

[15] P. Saha, R. Roy, A. K. Sarkar, and M. Pal, "Preferred time headway of drivers on two-lane highways with heterogeneous traffic," Transportation Letters, vol. 11, no. 4, pp. 200-207, 2019.

[16] J. Q. Leng, Y. Q. Feng, J. Zhai, L. Bao, and Y. He, “Travel time model of left-turning vehicles at signalized intersection," Mathematical Problems in Engineering, vol. 2012, pp. 1-10, Article ID 473847, 2012.

[17] J. J. Wu, H. J. Sun, and Z. Y. Gao, "Long-range correlations of density fluctuations in the Kerner-Klenov-Wolf cellular automata three-phase traffic flow model," Physical Review E, vol. 78, no. 3, pp. 1-5, 2008.

[18] T. R. Board, Highway Capacity Manual, Transportation Research Board, Washington, DC, USA, 2010.

[19] F. Lian, B. Chen, K. Zhang, L. Miao, J. Wu, and S. Luan, "Adaptive traffic signal control algorithms based on probe vehicle data," Journal of Intelligent Transportation Systems, vol. 1, pp. 1-17, 2020.

[20] E. Papapanagiotou and F. Busch, "Extended observer for urban traffic control based on limited measurements from connected vehicles," IEEE Transactions on Intelligent Transportation Systems, vol. 21, no. 4, pp. 1664-1676, 2020.

[21] $\mathrm{H} . \mathrm{Xu}, \mathrm{J}$. Chen, and J. Xu, "Integration of model-based signal control and queue spillover control for urban oversaturated signalized intersection," Mathematical Problems in Engineering, vol. 2019, pp. 1-15, Article ID 3149275, 2019.

[22] F. Zheng, J. Li, H. van Zuylen, X. Liu, and H. Yang, "Urban travel time reliability at different traffic conditions," Journal of Intelligent Transportation Systems, vol. 22, no. 2, pp. 106-120, 2017. 